\title{
Renormalizability of the massive Yang-Mills theory
}

\author{
S.A. Larin \\ Institute for Nuclear Research of the Russian Academy of Sciences, \\ 60th October Anniversary Prospect 7a, Moscow 117312, Russia
}

\begin{abstract}
It is shown that the massive Yang-Mills theory is on mass-shell renormalizable. Thus the Standard Model of electroweak interactions can be modified by removing terms with the scalar field from the Lagrangian in the unitary gauge. The resulting electroweak theory without the Higgs particle is on mass-shell renormalizable and unitary.
\end{abstract}


The massive Yang-Mills theory [1] is considered to be non-renormalizable [2], see also 3, 4] and references therein. The known way to get renormalizable and unitary theory with massive Yang-Mills bosons is due to the Higgs mechanism of spontaneous symmetry breaking [5]. The mechanism is used in the Standard $S U(2) \times U(1)$ Model of electroweak interactions [6] which is established to be renormalizable [7, see also 8 and references therein. In this way one introduces in the Model the scalar Higgs particle which one can hope to see in experiments.

The purpose of the present paper is to show that the massive Yang-Mills theory is in fact on mass-shell renormalizable. Hence the Standard Model can be modified by discarding from the Lagrangian in the unitary gauge all terms containing the scalar field.

Let us consider the massive Yang-Mills theory of gauge fields $W_{\mu}^{a}(x)$ defined by the generating functional of Green functions in the path integral form

$$
\begin{gathered}
Z(J)=\frac{1}{N} \int d W \exp \left(i \int d x\left(-\frac{1}{4} F_{\mu \nu}^{a} F_{\mu \nu}^{a}+\frac{1}{2} m^{2} W_{\mu}^{a} W_{\mu}^{a}+J_{\mu}^{a} W_{\mu}^{a}\right)\right) \\
F_{\mu \nu}^{a}=\partial_{\mu} W_{\nu}^{a}-\partial_{\nu} W_{\mu}^{a}+g f^{a b c} W_{\mu}^{b} W_{\nu}^{c}
\end{gathered}
$$

with the mass $m$ (in general with different masses $m_{a}$ ), $g$ is the coupling constant and $f^{a b c}$ are structure constants of some non-abelian group, e.g. the $S U(n)$ group.

The first term of the Lagrangian is invariant under the local gauge transformations

$$
W_{\mu}^{a} \rightarrow\left(W_{\mu}^{a}\right)^{\omega}=W_{\mu}^{a}+\partial_{\mu} \omega^{a}+g f^{a b c} W_{\mu}^{b} \omega^{c}+O\left(\omega^{2}\right)
$$

where $\omega^{a}(x)$ parametrize an element $\Omega(x)$ of the group in the usual way $\Omega(x)=\exp \left(i \omega^{a} T^{a}\right), T^{a}$ being generators of the corresponding Lie algebra.

The canonical quantization of this theory can cause some problems since $W_{0}^{a}$ are not canonical variables. But the perturbation theory can be developed since the field propagator is well defined

$$
D_{\mu \nu}^{a b}=-\frac{\delta^{a b}}{(2 \pi)^{4}} \frac{g_{\mu \nu}-k_{\mu} k_{\nu} / m^{2}}{k^{2}-m^{2}+i 0}
$$

It is the second term of the propagator which does not decrease at large $k$ and is assumed to violate renormalizability by power counting arguments. 
To establish on mass-shell renormalizability of the massive Yang-Mills theory (11) one should show that the S-matrix elements can be made finite by means of counterterms which can be absorbed into renormalization constants of the parameters $g$ and $m$ although the Green functions are divergent.

We will work within perturbation theory.

To regularize ultraviolet divergences we will use for convenience dimensional regularization [9] with the space-time dimension $d=4-2 \epsilon, \epsilon$ being the regularization parameter.

Let us consider the known model given by the initial $S U(2)$-invariant Lagrangian possessing the spontaneously broken symmetry

$$
L=-\frac{1}{4} F_{\mu \nu}^{a} F_{\mu \nu}^{a}+\left(D_{\mu} \Phi\right)^{+} D_{\mu} \Phi-\lambda\left(\Phi^{+} \Phi-v^{2}\right)^{2}
$$

with the doublet of scalar fields $\Phi(x)$ in the fundamental representation of the group.

Here $D_{\mu} \Phi=\left(\partial_{\mu}-i g \frac{\tau}{2} W_{\mu}^{a}\right) \Phi$ is the covariant derivative, $\tau^{a}$ are the Pauli matrices, $\lambda>0, v^{2}>0$.

To get the complete Lagrangian one makes the shift of the scalar field

$$
\Phi(x)=\frac{1}{\sqrt{2}}\left(\begin{array}{l}
i \phi_{1}(x)+\phi_{2}(x) \\
\sqrt{2} v+\chi(x)-i \phi_{3}(x)
\end{array}\right)
$$

fixes the gauge and adds ultraviolet counterterms.

Let us consider two gauges: the widely used $R_{\xi}$-gauge [7], [10] with an arbitrary parameter $\xi$ and the unitary gauge.

In the $R_{\xi}$-gauge one gets the theory described by the generating functional of Green functions

$$
\begin{gathered}
Z_{R_{\xi}}(J, K)=\frac{1}{N} \int d W d \phi d \chi d \bar{c} d c \exp \left(i \int d x\left(L_{R_{\xi}}+J_{\mu}^{a} W_{\mu}^{a}+K \chi\right)\right) \\
L_{R_{\xi}}=-\frac{1}{4} F_{\mu \nu}^{a} F_{\mu \nu}^{a}+\frac{m^{2}}{2} W_{\mu}^{a} W_{\mu}^{a}-m W_{\mu}^{a} \partial_{\mu} \phi^{a}+\frac{1}{2} \partial_{\mu} \phi^{a} \partial_{\mu} \phi^{a}+\frac{1}{2} \partial_{\mu} \chi \partial_{\mu} \chi \\
-\frac{M^{2}}{2} \chi^{2}+\frac{g}{2} W_{\mu}^{a}\left(\phi^{a} \partial_{\mu} \chi-\chi \partial_{\mu} \phi^{a}+\epsilon^{a b c} \phi^{b} \partial_{\mu} \phi^{c}\right)+\frac{m g}{2} \chi W_{\mu}^{a} W_{\mu}^{a} \\
+\frac{g^{2}}{8}\left(\chi^{2}+\phi^{a} \phi^{a}\right) W_{\mu}^{2}-\frac{g M^{2}}{4 m} \chi\left(\chi^{2}+\phi^{a} \phi^{a}\right)-\frac{g^{2} M^{2}}{32 m^{2}}\left(\chi^{2}+\phi^{a} \phi^{a}\right)^{2} \\
-\frac{1}{2 \xi}\left(\partial_{\mu} W_{\mu}^{a}+\xi m \phi^{a}\right)^{2}
\end{gathered}
$$




$$
\begin{gathered}
+\partial_{\mu} \bar{c}^{a}\left(\partial_{\mu} c^{a}-g \epsilon^{a b c} c^{b} W_{\mu}^{c}\right) \\
-\xi m^{2} \bar{c}^{a} c^{a}-\frac{g}{2} \xi m \chi \bar{c}^{a} c^{a}+\frac{g}{2} \xi m \epsilon^{a b c} \bar{c}^{a} c^{b} \phi^{c} \\
+ \text { counterterms }
\end{gathered}
$$

This theory describes three physical massive vector bosons with the mass $m=g v / \sqrt{2}$, and the physical Higgs field $\chi$ with the mass $M=2 \lambda v$. Here are also Goldstone ghosts $\phi^{a}$ and Faddeev-Popov ghosts $c^{a}$ with masses $\xi m^{2}$. The structure of the counterterms (consistent with gauge invariance and Slavnov-Taylor identities [11, 12] to ensure unitarity) is well known, see e.g. 8].

This is the renormalizable gauge, i.e. Green functions are finite.

In the unitary gauge defined by the condition $\phi^{a}=0$ one has the Lagrangian

$$
\begin{gathered}
L_{U}=-\frac{1}{4} F_{\mu \nu}^{a} F_{\mu \nu}^{a}+\frac{m^{2}}{2} W_{\mu}^{a} W_{\mu}^{a}+\frac{1}{2} \partial_{\mu} \chi \partial_{\mu} \chi-\frac{M^{2}}{2} \chi^{2} \\
+\frac{m g}{2} \chi W_{\mu}^{a} W_{\mu}^{a}+\frac{g^{2}}{8} \chi^{2} W_{\mu}^{a} W_{\mu}^{a}-\frac{g M^{2}}{4 m} \chi^{3}-\frac{g^{2} M^{2}}{32 m^{2}} \chi^{4}+\text { counterterms }
\end{gathered}
$$

The theory in the unitary gauge is renormalizable only on mass-shell, i.e. Green functions are divergent at $\epsilon \rightarrow 0$ but the S-matrix elements are finite. In this gauge all unphysical particles (longitudinal quanta of vector fields and ghosts) are absent and unitarity of the theory is manifest.

To show equivalence of S-matrix elements in two gauges one uses the functional integral technique. Let us repeat it for the case of the Landau gauge $\xi=0$ (for simplicity) which corresponds in fact to the Lorentz gauge $\partial_{\mu} W_{\mu}^{a}=0$. The generating functional of Green functions in the $L$-gauge is

$Z_{L}(J, K)=\frac{1}{N} \int d W d \phi d \chi \exp \left(i \int d x\left(L_{R}+J_{\mu}^{a} W_{\mu}^{a}+K \chi\right)\right) \Delta_{L}(W) \delta\left(\partial_{\mu} W_{\mu}\right)$

where $\Delta_{L}(W)$ is the Faddeev-Popov determinant [13 and $L_{R}$ is obtained from $L_{R_{\xi}}$ by omitting terms depending on $\xi$ and $c^{a}$ (and by corresponding modification of counterterms). The Lagrangian $L_{R}$ is invariant under the following gauge transformations

$$
\begin{gathered}
W_{\mu}^{a} \rightarrow\left(W_{\mu}^{\omega}\right)^{a}=W_{\mu}^{a}+\partial_{\mu} \omega^{a}+\tilde{g} f^{a b c} W_{\mu}^{b} \omega^{c}+O\left(\omega^{2}\right) \\
\phi^{a} \rightarrow\left(\phi^{\omega}\right)^{a}=\phi^{a}-\tilde{m} \omega^{a}-\frac{\tilde{g}}{2} f^{a b c} \phi^{b} \omega^{c}-\frac{\tilde{g}}{2} \chi \omega^{a}+O\left(\omega^{2}\right)
\end{gathered}
$$




$$
\chi \rightarrow \chi^{\omega}=\chi-\frac{\tilde{g}}{2} \phi^{a} \omega^{a}+O\left(\omega^{2}\right)
$$

where

$$
\tilde{g}=\frac{z_{1}}{z_{2}} g \quad \tilde{m}=\frac{z_{1}}{z_{2}} m
$$

and $z_{1}, z_{2}$ are renormalization constants of the triple $W$-vertex and the $W$ field.

One inserts in the functional integral the unity

$$
\Delta_{U}(\chi) \int d w \delta\left(\phi^{\omega}\right)=1
$$

Making the known change of variables

$$
W_{\mu} \rightarrow W_{\mu}^{\omega^{-1}}, \quad \phi \rightarrow \phi^{\omega^{-1}}, \quad \chi \rightarrow \chi^{\omega^{-1}}, \quad \omega^{-1} \rightarrow \omega
$$

and integrating over $\omega$ one obtains

$$
Z_{L}(J, K)=\frac{1}{N} \int d W d \phi d \chi \exp \left(i \int d x\left(L_{U}+J_{\mu} W_{\mu}^{\tilde{\omega}}+K \chi^{\tilde{\omega}}\right)\right) \Delta_{U}(\chi) \delta(\phi)
$$

where $\tilde{\omega}$ is defined from the equation

$$
\partial_{\mu}\left(W_{\mu}^{\tilde{\omega}}\right)^{a}=\partial_{\mu}\left(W_{\mu}^{a}+\partial_{\mu} \tilde{\omega}^{a}+\tilde{g}\left(f^{a b c} W_{\mu}^{b} \tilde{\omega}^{c}\right)\right)+O\left(\tilde{\omega}^{2}\right)=0
$$

The Lagrangian $L_{U}$ is given in eq.(15).

The functional $\Delta_{U}(\chi)$ can be presented on the surface $\phi^{a}=0$ as

$$
\Delta_{U}(\chi)=\operatorname{det}\left|\tilde{m}+\frac{\tilde{g}}{2} \chi(x)\right|^{3}=\text { const } \cdot \exp \left(\int \delta^{d}(0) \ln \left(1+\frac{g}{2 m} \chi(x)\right)^{3} d x\right)
$$

In dimensional regularization this functional is just a constant and can be absorbed in the normalization factor $N$ although this simplification is not essential for the following derivation.

One obtaines

$$
Z_{L}(J, K)=\frac{1}{N} \int d W d \chi \exp \left(i \int d x\left(L_{U}+J_{\mu} W_{\mu}^{\tilde{\omega}}+K \chi^{\tilde{\omega}}\right)\right)
$$

The expression (11) differs from the generating functional of Green functions in the unitary gauge

$$
Z_{U}(J, K)=\frac{1}{N} \int d W d \chi \exp \left(i \int d x\left(L_{U}+J_{\mu} W_{\mu}+K \chi\right)\right)
$$


only by source terms. It is known that this difference is not essential for the S-matrix elements, see e.g. 8]. Thus the physical equivalence of the L-gauge and the U-gauge is proved.

From eq. (111) one sees that the counterterms of $L_{U}$ are given by the counterterms of $L_{R}$ at $\phi^{a}(x)=0$.

To consider renormalization for our purpose it is convenient to use the Bogoliubov-Parasiuk-Hepp subtraction scheme [14. As it is well known in this scheme a counterterm of e.g. a primitively divergent Feynman diagram is the truncated Taylor expansion of the diagram itself at some fixed values of external momenta. Hence counterterms of mass dependent diagrams are also mass dependent.

Let us now analyze the dependence of the Green functions on the Higgs mass $M$. We will use for this purpose the expansion in large $M$. The algorithm for the large mass expansion of Feynman diagrams is given in [15], it can be rigorously derived e.g. with the technique of [16].

The representation (11) ensures for the regularized Green functions of the fields $W$ and $\chi$ that the large $M$-expansion of $M$-dependent contributions contain either terms with integer negative powers of $M^{2}$ or terms with noninteger powers of $M^{2}$ (non-integer powers contain $\epsilon$ ). This is because each vertex with the factor $M^{2}$ has three or four attached $\chi$-lines due to the structure of $L_{U}$. Corresponding counterterms (i.e. counterterms relevant for $L_{U}$ ) have the same property within the large- $M$ expansion. (In contrast, counterterms of e.g. the four- $\phi$ vertex in $L_{R}$ contain polynomial in $M$ terms because of the $M^{2}$-factors in the couplings of $L_{R}$.)

Let us further consider Green functions with external $W$-bosons only. We will first shortly formulate the result. The eq.(11) ensures that if one removes from a renormalized Green function $M$-dependent terms then the remaining part is finite. On the Lagrangian level it means that one removes from $L_{U}$ all terms containing the field $\chi$. Thus one obtains the theory

$$
\begin{gathered}
\tilde{Z}(J)=\frac{1}{N} \int d W \exp \left(i \int d x\left(L_{Y M}+J_{\mu} W_{\mu}^{\tilde{\omega}}\right)\right) \\
L_{Y M}=-\frac{1}{4} F_{\mu \nu}^{a} F_{\mu \nu}^{a}+m^{2} W_{\mu}^{a} W_{\mu}^{a}+\text { counterterms }
\end{gathered}
$$

with finite off-shell Green functions, where $W_{\mu}^{\tilde{\omega}}$ is given by (10). Since the difference between $W_{\mu}^{\tilde{\omega}}$ and $W_{\mu}$ in the source term is not essential for $S$-matrix elements the massive Yang-Mills theory is renormalizable on mass-shell. 
Let us elaborate these arguments in more detail. The representation (III) ensures, see e.g. [8], that the following on mass-shell expressions for the renormalized Green functions (relevant for the S-matrix elements) should coincide

$$
\begin{gathered}
\left.\left(\frac{1}{\sqrt{z}}\right)^{n} \prod_{i=1}^{n}\left(k_{i}^{2}-m^{2}\right) G_{\mu_{1} \ldots \nu_{n}}^{a_{1} \ldots a_{n}}\left(k_{1} \ldots k_{n}\right)\right|_{L-\text { gauge }} \stackrel{k_{i}^{2}=m^{2}}{=} \\
\left.\left(\frac{1}{\sqrt{z}}\right)^{n} \prod_{i=1}^{n}\left(k_{i}^{2}-m^{2}\right) G_{\mu_{1} \ldots \nu_{n}}^{a_{1} \ldots a_{n}}\left(k_{1} \ldots k_{n}\right)\right|_{U-\text { gauge }}
\end{gathered}
$$

where $z$ is the residue of the propagator pole

$\delta^{a b}\left(g_{\mu \nu}-\frac{k_{\mu} k_{\nu}}{k^{2}}\right)^{2} z=\left.\lim _{k^{2} \rightarrow m^{2}}\left(k^{2}-m^{2}\right)\left(g_{\mu \nu}-\frac{k_{\mu} k_{\nu}}{k^{2}}\right) \int e^{i k x} \frac{\delta^{2} Z(J)}{\delta J_{\mu}^{a}(x) J_{\nu}^{b}(0)} d x\right|_{J=0}$

We apply again the large- $M$ expansion to both sides of eq.(14) before removing regularization. In the L-gauge one can present renormalization in a standard form of the $R$-operation for individual diagrams. This ensures that $M$-dependent terms in the large $M$-expansion of the l.h.s. of (14) are finite at $\epsilon \rightarrow 0$ separately from $M$-independent terms. Hence in the r.h.s. $M$-dependent terms are also finite separately from $M$-independent terms. Thus if one removes all $M$-dependent terms from the r.h.s. of (14) one is left with a finite expression. On the Lagrangian level it means in the unitary gauge that one removes from $L_{U}$ all terms containing the field $\chi$ and also all $M$-dependent terms in the large- $M$ expansion of counterterms. The resulting theory is on mass-shell finite. This is the massive Yang-Mills theory

$$
\begin{gathered}
Z(J)=\frac{1}{N} \int d W \exp \left(i \int d x\left(L_{Y M}+J_{\mu}^{a} W_{\mu}^{a}\right)\right) \\
L_{Y M}=-\frac{1}{4} z_{2}\left(\partial_{\mu} W_{\nu}^{a}-\partial_{\nu} W_{\mu}^{a}+\frac{z_{1}}{z_{2}} g f^{a b c} W_{\mu}^{b} W_{\nu}^{c}\right)^{2}+z_{m} m^{2} W_{\mu}^{a} W_{\mu}^{a}
\end{gathered}
$$

After renormalizability is established one can fix renormalization constants $z_{1}, z_{2}$ and $z_{m}$ within the theory (16) (without referring to the L-gauge) by proper normalization conditions.

It is known that the Higgs theories of vector mesons posses so called tree level unitarity, see e.g. [3] and references therein. Tree level cross sections of such theories grow at high energies slowly enough and do not exceed the 
so called unitary limit imposed by the unitarity condition. The reversed statement is also proved: from the condition of tree level unitarity follows that a theory of vector mesons should be a Higgs theory [17. But one can see that tree level unitarity is not the necessary condition for renormalizability. Tree level unitarity is violated in the massive Yang-Mills theory. It indicates that higher order contributions become relevant at high energies.

The above derivation of on mass-shell renormalizability is applicable also to other gauge groups. It can be straightforwardly applied to the Standard $S U(2) \times U(1)$ Model of electroweak interactions. The presence of the $U(1)$ gauge boson and of fermions does not change the derivation. One can remove from the Lagrangian in the unitary gauge all terms containing the scalar field. The resulting electroweak theory without the Higgs particle is on mass-shell renormalizable and unitary.

The author is grateful to D.S. Gorbunov and S.M. Sibiryakov for helpful discussions.

\section{References}

[1] C.N. Yang and R.L. Mills, Phys. Rev. 96 (1954) 191.

[2] D.G. Boulware, Ann. of Phys. 56 (1970) 140.

[3] J.C. Taylor, Gauge theories of weak interactions, Cambridge University Press, 1976.

[4] C. Itzykson and J.B. Zuber, Quantum field theory, New York, Mcgrawhill, 1980.

[5] P.W. Higgs, Phys. Lett. 12 (1964) 132.

F.Englert and R. Brout, Phys. Rev. Lett. 13 (1964) 321.

T.W.B. Kibble, Phys. Rev. 155 (1967) 1554.

[6] S.L. Glashow, Nucl. Phys. 22 (1961) 579.

S. Weinberg, Phys. Rev. Lett. 19 (1967) 264.

A. Salam, in Elementary Particle Theory, ed. N. Svartholm, Stochholm, Almquist and Wiksell, 1968. 
[7] G. 't Hooft, Nucl. Phys. B 35 (1971) 167.

[8] L.D. Faddeev and A.A. Slavnov, Gauge fields. Introduction to quantum theory, Front. Phys. 83 (1990) 1.

[9] K.G. Wilson and M.E. Fisher, Phys. Rev. Lett. 28 (1972) 240.

G. 't Hooft and M. Veltman, Nucl.Phys. B 44 (1972) 189.

C.G. Bollini and J.J. Giambiagi, Phys. Lett. B 40 (1972) 566.

J.F. Ashmore, Nuovo Cimento Lett. 4 (1972) 289.

G.M. Cicuta and E. Montaldi, Nuovo Cimento Lett. 4 (1972) 329.

[10] K. Fujikawa, B.W. Lee and A.I. Sanda, Phys. Rev. D6 (1972) 2923.

[11] A.A. Slavnov, Theor. Math. Phys. 10 (1972) 99.

[12] J.C. Taylor, Nucl. Phys. B33 (1971) 436.

[13] L.D. Faddeev and V.N. Popov, Phys. Lett. B 25 (1967) 30.

[14] N.N. Bogoliubov and O.S. Parasiuk, Acta Math. 97 (1957) 227.

K. Hepp, Comm. Math. Phys. 2 (1966) 301.

[15] S.A. Larin, T. van Ritbergen and J.A.M. Vermaseren, Nucl. Phys. B 438 (1995) 278.

[16] S.A. Larin, Phys. Lett. B 469 (1999) 220.

[17] J.M. Cornwall, D.N. Levin and G. Tiktopoulos, Phys. Rev. D 10 (1974) 1145. 